\title{
Supporting Information for: Universality of Entropic Surface Segregation from Athermal Polymer Blends Due to Conformational Asymmetry
}

\author{
Russell K. W. Spencer ${ }^{*, \dagger}$ and Mark W. Matsen*,† \\ ${ }^{\dagger}$ Institut für Theoretische Physik, Georg-August Universität, Friedrich-Hund-Platz 1, 370r7 \\ Göttingen, Germany \\ ¥Department of Chemical Engineering, Department of Physics \& Astronomy, and Waterloo \\ Institute for Nanotechnology, University of Waterloo, Waterloo, Ontario N2L 3G1, Canada
}

\section{Previous Simulations by Kumar et al.:}

In order to explicitly illustrate the inaccuracies in the previous MC simulations of Kumar and coworkers [1], we repeat their four simulations listed in Table SI. For this, we switch to their bending energy,

$$
\frac{E_{\mathrm{bend}}}{k_{B} T}=\frac{\kappa}{2} \sum_{j=1}^{n_{s}} \sum_{i=1}^{N-1}\left(1-\mathbf{u}_{i}^{(j)} \cdot \mathbf{u}_{i+1}^{(j)}\right)^{2}
$$

Kumar et al. equilibrated their systems until there are no overlaps between the monomers and then collected statistics for approximately $10^{5} \mathrm{MC}$ steps per monomer. We, on the other hand, equilibrated the four systems for approximately $10^{9} \mathrm{MC}$ steps per monomer, and collected statistics over a similar duration. Even this was insufficient for the $N=500$ chains considered by Kumar et al., which is why we excluded them from our comparison.

Table SI: Parameters for the simulations repeated from ref [1]

\begin{tabular}{ccccccc}
\hline$N$ & $\kappa$ & $n_{s}$ & $n_{f}$ & $L_{x} \times L_{y} \times L_{z}$ & $\bar{R}_{g}$ & $\gamma$ \\
\hline 10 & 1.0 & 50 & 50 & $9.4 \times 9.4 \times 18$ & 1.67 & 0.16 \\
25 & 1.0 & 20 & 20 & $9.4 \times 9.4 \times 18$ & 2.89 & 0.18 \\
50 & 1.0 & 10 & 10 & $9.4 \times 9.4 \times 18$ & 4.21 & 0.17 \\
100 & 1.0 & 5 & 5 & $9.4 \times 9.4 \times 18$ & 5.92 & 0.16 \\
\hline
\end{tabular}

Inaccuracies in the previous simulations are immediately evident from the conformational asymmetry parameter, $\gamma=R_{g, s} / R_{g, f}-1$, which we now express in terms of radii of gyration to coincide with the definition used by Kumar et al. Our estimates listed in Table SI are considerably different from their estimates: $\gamma=0.14,0.17,0.06$, and 0.10 for $N=10,25,50$, and 100, respectively. As expected, our values of $\gamma$ are relatively independent of $N$, as they should be given that $R_{g, \alpha} \rightarrow a_{\alpha} \sqrt{N / 6}$ for $N \rightarrow \infty$. In contrast, their $\gamma$ values exhibit an erratic dependence on $N$, signifying that something must be seriously wrong. The fact that 
their values of $\gamma$ are consistently smaller than ours implies that part of the problem is due to insufficient equilibration. Given that the inaccuracy is most serious for the larger polymers, we conclude that they did not allow sufficient time for their stiff polymers to evolve from the random walks they were initialized with to the more extended conformations corresponding to equilibrium.

Further problems become apparent when we examine the distributions of monomer centers, $\rho_{f}(z)$ and $\rho_{s}(z)$. Our improved results are shown in Figure S1 from the $z=0$ wall to the center of the simulation box at $z=9$. As discussed in the main paper, our simulation results are accurate to within the width of the curves. The insets plot the concentration difference

$$
\Delta \rho(z) \equiv \rho_{s}(z)-\rho_{f}(z)
$$

plotted by Kumar et al. Also included in the insets are fits to $\Delta \rho(z)=A_{\rho} F_{\rho}(Z)+$ constant, where the constant just accounts for the fact that $\Delta \rho(z) \neq 0$ in the bulk. The fits agree well with the simulations for $N=10$ and 25, apart from the short-range oscillations next to the wall. Although they start to fail for $N=50$, this is simply because the simulation boxes used by Kumar et al. were too small to reach bulk conditions, which is why we have not included a fit for $N=100$.
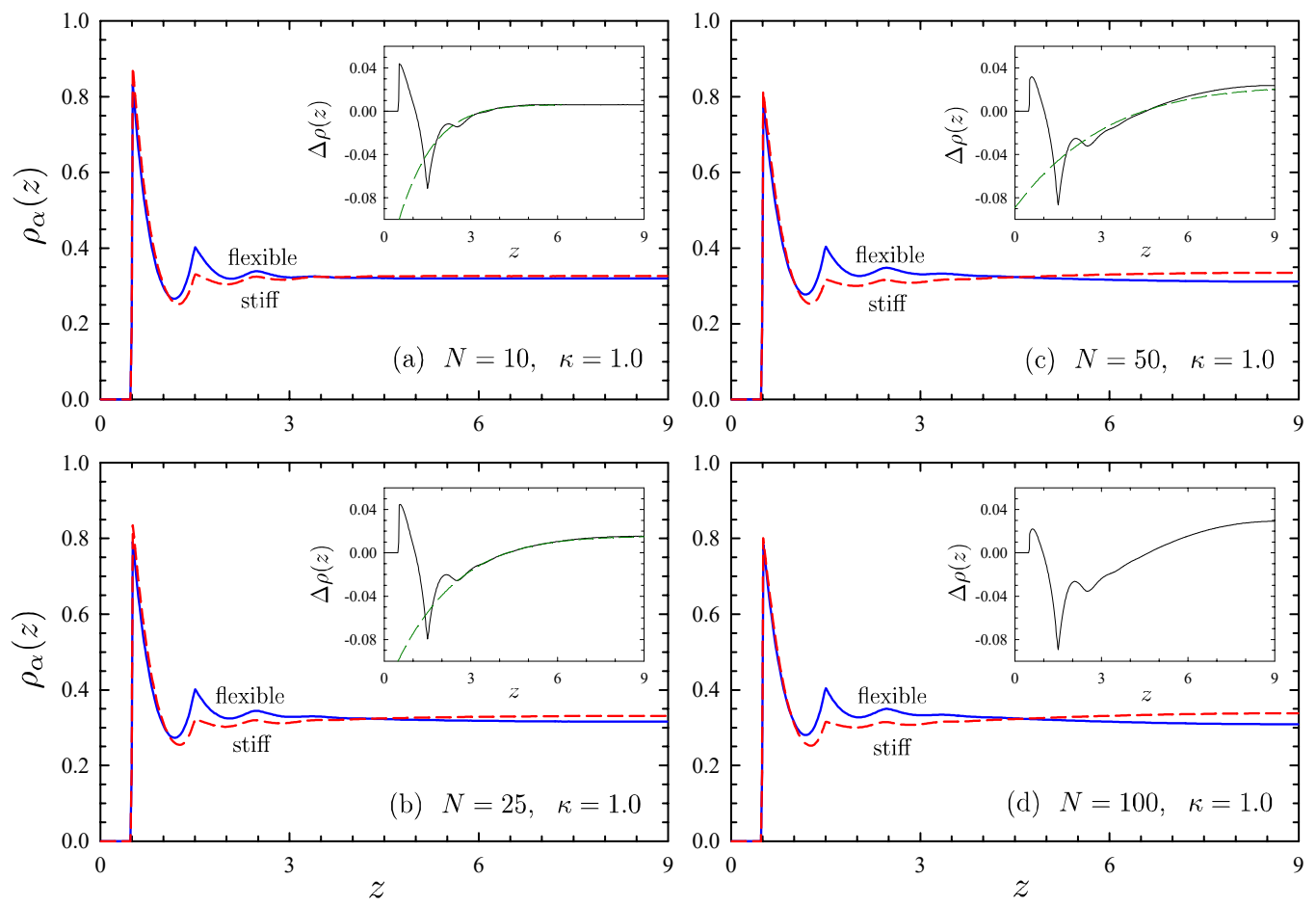

Figure S1: Distribution of monomer centers from flexible polymers, $\rho_{f}(z)$ (solid curves), and from stiff polymers, $\rho_{s}(z)$ (dashed curves), next to a hard wall, evaluated for the four systems listed in Table SI. The insets plot $\Delta \rho(z)$ (solid curves) and fits to the universal profile, $F_{\rho}(Z)$ (dashed curves). 
To illustrate the inaccuracy in the previous results of Kumar et al., Figure S2 overlays the equivalent simulation from data from Figure 3 of ref [1]. There are clear discrepancies in all four cases, including the short polymers of $N=10$. Note that our plots extend from the wall (i.e., $z=0$ ) to the middle of the simulation box (i.e., $z=L_{z} / 2$ ), whereas their plots only extend from $z=0.5$ to $z=3.0$ because they concluded that $\rho_{f}(z) \approx \rho_{s}(z)$ for $z \gtrsim 3.0$. However, this cannot actually be true since the conservation of material requires the integral of $\Delta \rho(z)$ to be zero, irregardless of any statistical noise. In our simulations, for example, there is a significant excess of flexible polymers at the wall, which in turn causes an enrichment of stiff polymers in the bulk region. In their simulations, on the other hand, the excess of stiff polymers at the surface must be balanced by an enrichment of flexible polymers in the bulk. They presumably failed to notice this effect due to the large statistical noise in their simulations.
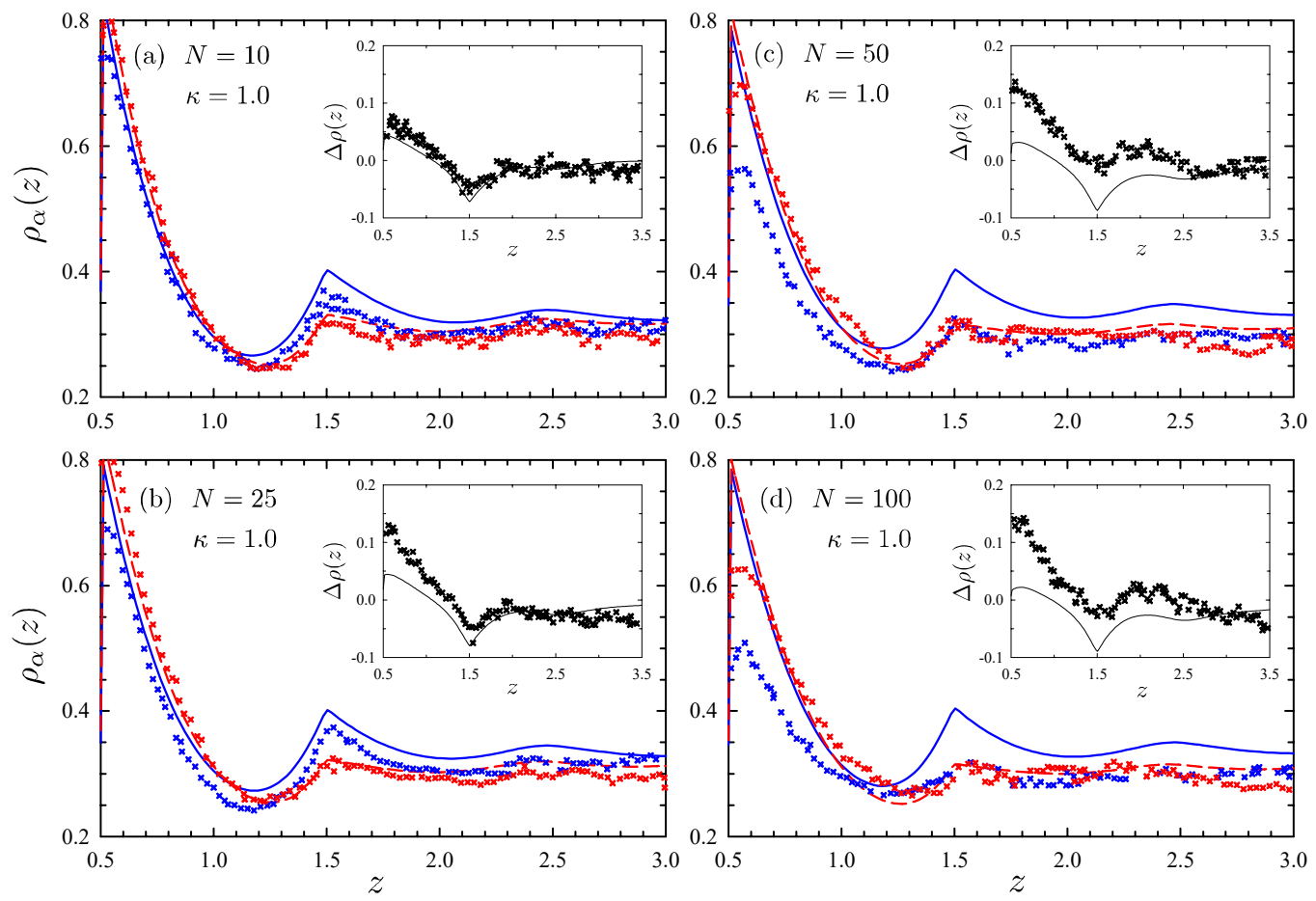

Figure S2: Curves from Figure S1 overlayed with data from Ref. [1] (symbols). The range of the scales has been adjusted to match that of Ref. [1].

\section{Calculations by Donley et al.:}

Donley et al. [2] attempted to reconcile the slight segregation of stiff polymers predicted by the PRISM calculations and MC simulations of Kumar et al. [1, 3] with the significant segregation of flexible polymers predicted by the SCFT calculations of Wu et al. [4] and 
observed in the experiments of Sikka et al. [5,6]. They did so by assuming that the appropriate self-consistent fields for the flexible (A) and stiff (B) polymers of the MC simulations were

$$
\begin{aligned}
& w_{A}(\mathbf{r})=-\hat{c}_{A A}(0) \Delta \rho_{A}(\mathbf{r})-\hat{c}_{A B}(0) \Delta \rho_{B}(\mathbf{r}) \\
& w_{B}(\mathbf{r})=-\hat{c}_{A B}(0) \Delta \rho_{A}(\mathbf{r})-\hat{c}_{B B}(0) \Delta \rho_{B}(\mathbf{r})
\end{aligned}
$$

where the $\hat{c}_{\alpha \beta}(0)$ values can be obtained from the direct correlation functions of PRISM and $\Delta \rho_{\alpha}(\mathbf{r}) \equiv \rho_{\alpha}(\mathbf{r})-\bar{\rho}_{\alpha}$ are deviations of monomer density from their bulk values. They then provided two distinct arguments why this would lead a negligible surface segregation.

The first argument in Section III, General Treatment of Polymer Blends, of Donley et al. relies on mapping eqs (S3) and (S4) onto the Helfand-Tagami [7] SCFT field equations

$$
\begin{aligned}
& w_{A}(\mathbf{r})=\chi \frac{\Delta \rho_{B}(\mathbf{r})}{\bar{\rho}_{0}}+\zeta \frac{\Delta \rho_{A}(\mathbf{r})+\Delta \rho_{B}(\mathbf{r})}{\bar{\rho}_{0}} \\
& w_{B}(\mathbf{r})=\chi \frac{\Delta \rho_{A}(\mathbf{r})}{\bar{\rho}_{0}}+\zeta \frac{\Delta \rho_{A}(\mathbf{r})+\Delta \rho_{B}(\mathbf{r})}{\bar{\rho}_{0}}
\end{aligned}
$$

where the interaction parameters, $\chi$ and $\zeta$, are defined based on the reference density $\bar{\rho}_{0}=$ $\bar{\rho}_{A}+\bar{\rho}_{B}$. It is important to note that these field equations assume the A and B monomers occupy equal volumes of $1 / \bar{\rho}_{0}$. If one tries to directly map eqs (S3) and (S4) onto eqs (S5) and (S6), it becomes immediately apparent that

$$
\frac{\zeta}{\bar{\rho}_{0}}=-\hat{c}_{A A}(0)=-\hat{c}_{B B}(0)
$$

which contradicts the PRISM calculations.

In an attempt to avoid this restriction, Donley et al. scaled the size of the B monomers by a factor of

$$
\lambda=\sqrt{\hat{c}_{B B}(0) / \hat{c}_{A A}(0)}
$$

The effect of the scaling on various quantities is listed in eq (11) of Donley et al. They did not explicitly mention the effect on the fields, but it is easy to show that $w_{A}^{\prime}(\mathbf{r})=w_{A}(\mathbf{r})$ and $w_{B}^{\prime}(\mathbf{r})=w_{B}(\mathbf{r}) / \lambda$. In terms of scaled quantities, eqs (S3) and (S4) transform to

$$
\begin{aligned}
& w_{A}^{\prime}(\mathbf{r})=\chi^{\prime} \frac{\Delta \rho_{B}^{\prime}(\mathbf{r})}{\bar{\rho}_{0}^{\prime}}+\zeta^{\prime} \frac{\Delta \rho_{A}(\mathbf{r})+\Delta \rho_{B}^{\prime}(\mathbf{r})}{\bar{\rho}_{0}^{\prime}} \\
& w_{B}^{\prime}(\mathbf{r})=\chi^{\prime} \frac{\Delta \rho_{A}(\mathbf{r})}{\bar{\rho}_{0}^{\prime}}+\zeta^{\prime} \frac{\Delta \rho_{A}(\mathbf{r})+\Delta \rho_{B}^{\prime}(\mathbf{r})}{\bar{\rho}_{0}^{\prime}}
\end{aligned}
$$

where the interaction parameters

$$
\begin{gathered}
\chi^{\prime}=\frac{\bar{\rho}_{0}^{\prime}}{2}\left(\hat{c}_{A A}(0)+\hat{c}_{B B}^{\prime}(0)-2 \hat{c}_{A B}^{\prime}(0)\right) \\
\zeta^{\prime}=-\frac{\bar{\rho}_{0}^{\prime}}{2}\left(\hat{c}_{A A}(0)+\hat{c}_{B B}^{\prime}(0)\right)
\end{gathered}
$$

are now defined in terms of the reference density $\bar{\rho}_{0}^{\prime}=\bar{\rho}_{A}+\bar{\rho}_{B}^{\prime}$. Although eqs (S9) and (S10) have the same form as the Helfand-Tagami fields in eqs (S5) and (S6), they are for unequal monomer sizes whereas the Helfand-Tagami equations assume monomers of equal size. 
If one scales the fields in eqs (S3) and (S4), then the same needs to be done for the Helfand-Tagami fields in eqs (S5) and (S6) before making a comparison. Naturally, the scaling cannot change the end result. To illustrate this point, we now scale the HelfandTagami field equations, which gives

$$
\begin{aligned}
w_{A}^{\prime}(\mathbf{r}) & =\chi \frac{\Delta \rho_{B}^{\prime}(\mathbf{r}) / \lambda}{\bar{\rho}_{0}}+\zeta \frac{\Delta \rho_{A}(\mathbf{r})+\Delta \rho_{B}^{\prime}(\mathbf{r}) / \lambda}{\bar{\rho}_{0}} \\
w_{B}^{\prime}(\mathbf{r}) & =\frac{\chi}{\lambda} \frac{\Delta \rho_{A}(\mathbf{r})}{\bar{\rho}_{0}}+\frac{\zeta}{\lambda} \frac{\Delta \rho_{A}(\mathbf{r})+\Delta \rho_{B}^{\prime}(\mathbf{r}) / \lambda}{\bar{\rho}_{0}}
\end{aligned}
$$

Matching the coefficients of $\Delta \rho_{A}(\mathbf{r})$ in eqs (S9) and (S13) and the coefficients of $\Delta \rho_{B}^{\prime}(\mathbf{r})$ in eqs (S10) and (S14) gives

$$
\frac{\zeta^{\prime}}{\bar{\rho}_{0}^{\prime}}=\frac{\zeta}{\bar{\rho}_{0}}=\frac{\zeta}{\lambda^{2} \bar{\rho}_{0}}
$$

which is only possible if $\lambda=1$ or equivalently if $\hat{c}_{A A}(0)=\hat{c}_{B B}(0)$ as concluded previously in eq (S7). The fact that their field equations cannot, in general, be mapped onto the Helfand-Tagami field equations invalidates the remainder of their first argument.

The second argument in Section IV, Athermal Blends, of Donley et al. uses the PRISM prediction

$$
\hat{c}_{A B}(0)=\gamma^{2} \hat{c}_{A A}(0)=\frac{1}{\gamma^{2}} \hat{c}_{B B}(0)
$$

where $\gamma=b_{B} / b_{A}>1$ is the ratio of statistical segment lengths of the stiff and flexible polymers. By inserting these relationships into eqs (S3) and (S4), they obtained

$$
\begin{aligned}
& w_{A}(\mathbf{r})=\frac{1}{\gamma} \sqrt{\hat{c}_{A A}(0) \hat{c}_{A B}(0)} \Delta \rho_{A}(\mathbf{r})+\frac{1}{\gamma} \sqrt{\hat{c}_{B B}(0) \hat{c}_{A B}(0)} \Delta \rho_{B}(\mathbf{r}) \\
& w_{B}(\mathbf{r})=\gamma \sqrt{\hat{c}_{A A}(0) \hat{c}_{A B}(0)} \Delta \rho_{A}(\mathbf{r})+\gamma \sqrt{\hat{c}_{B B}(0) \hat{c}_{A B}(0)} \Delta \rho_{B}(\mathbf{r})
\end{aligned}
$$

which implies that the force acting on $\mathrm{B}$ monomers is $\gamma^{2}$ times stronger than the force acting on A monomers. Donley et al. then reduced the size of the A monomers by a factor of $\gamma$ and increased the size of the B monomers by the same factor $\gamma$, obtaining a system where the $\mathrm{A}$ and $\mathrm{B}$ monomers both have equal statistical lengths

$$
b_{A}^{\prime}=b_{B}^{\prime}=\sqrt{b_{A} b_{B}}
$$

and equal forces

$$
w_{A}^{\prime}(\mathbf{r})=w_{B}^{\prime}(\mathbf{r})=\sqrt{\hat{c}_{A A}(0) \hat{c}_{A B}(0)} \Delta \rho_{A}(\mathbf{r})+\sqrt{\hat{c}_{B B}(0) \hat{c}_{A B}(0)} \Delta \rho_{B}(\mathbf{r})
$$

On this basis, Donley et al. argued that the behavior of the two polymers should be equivalent, but this argument is also flawed.

The scaling transforms the system from polymers of equal polymerizations (i.e., $N_{A}=$ $N_{B}$ ) to polymers of unequal polymerizations (i.e., $N_{A}^{\prime}=N_{B}^{\prime} / \gamma^{2}$ ). Given this asymmetry in polymerization, there is no reason to expect the segregation to be negligible. Again, the scaling cannot possibly alter the behavior. Prior to the scaling, the average end-toend length of the $\mathrm{B}$ polymers was greater than that of the A polymers because $b_{B}>b_{A}$ and the net force acting on the $\mathrm{B}$ polymers was stronger than for the A polymers because $\left|w_{B}(\mathbf{r})\right|>\left|w_{A}(\mathbf{r})\right|$. This remains just as true after the scaling. Although the scaling results in $\mathrm{A}$ and $\mathrm{B}$ monomers with equal statistical lengths and equal forces, the B polymers still have a larger average end-to-end length and experience a stronger net force because $N_{B}^{\prime}>N_{A}^{\prime}$. 


\section{References}

[1] Kumar, S. K.; Yethiraj, A.; Schweizer, K. S.; Leermakers, F. A. M. The effects of local stiffness disparity on the surface segregation from binary polymer blends. J. Chem. Phys. 1995, 103, 10332-10346.

[2] Donley, J. P.; Wu, D. T.; Fredrickson, G. H. On the Control of Surface Enrichment in Polymer Blends and Copolymers. Macromolecules 1997 30, 2167-2174.

[3] Yethiraj, A.; Kumar, S.; Hariharan, A.; Schweizer, K. S. Surface segregation in polymer blends due to stiffness disparity. J. Chem. Phys. 1994, 100, 4691-4694.

[4] Wu, D. T.; Fredrickson, G. H.; Carton, J.-P. Surface Segregation in Conformationally Asymmetric Polymer Blends: Incompressibility and Boundary Conditions. J. Chem. Phys. 1996, 104, 6387-6397.

[5] Sikka, M.; Singh, N.; Karim, A.; Bates, F. S.; Satija, S. K.; Majkrzak, C. F. Entropydriven surface segregation in block copolymer melts. Phys. Rev. Lett. 1993, 70, 307-310.

[6] Sikka, M.; Singh, N.; Bates, F. S.; Karim, A.; Satija, S.; Majkrzak, C. F. Surface segregation in model symmetric polyolefin diblock copolymer melts. J. Phys. II France 1994, 4, 2231-2248.

[7] Helfand, E.; Tagami, Y. Theory of the Interface between Immiscible Polymers. II $J$. Chem. Phys. 1972, 56, 3592-3601. 